\section{Gianluca Bossi}

The microenvironment of solid tumors is composed of malignant cells surrounded by a reactive stroma containing extracellular matrix with a huge infiltration of non-malignant populations (myeloid cells, lymphocytes, fibroblast, endothelial cells), which together with several cytokines/chemokines, tissue remodelling and angiogenesis support an inflammatory milieu. Tumor growth and metastasis are the result of a complex bidirectional interaction between cells that progressively acquire molecular alterations, and a transformed phenotype (cell-autonomous functions) and the surrounding host cells (non-cell-autonomous functions).

The TP53 gene is mutated in about half of all human cancers. Missense mutations are the most prevalent alterations (75\%) located mainly within the DNA binding domain. Most of these alterations hold the fulllength protein, often present in grossly elevated levels compared to the wild type (wt) p53 in normal cells, that lose the tumor suppressor functions (loss-of-function, LOF), and acquire novel functions (gain-of-function, GOF) through which contribute to tumorigenesis, tumor progression and chemo- or radiotherapy resistance. In recent years many cell-autonomous GOFs mutant (mut) p53 have been described, mostly linked to the ability of mutated proteins to control the expression of specific target genes [1]. Noteworthy, emerging evidences uncovered the existence of non-cell-autonomous wtp53 functions by promoting anti-tumor microenvironment $[2,3]$, whereas barely reported the non-cell-autonomous GOFs mutp53 [4].

We previously demonstrated that inducible depletion in vivo of endogenous mutp53 reduces tumor growth, stromal invasion, and angiogenesis in xenografted HT29 colon cancer cells [5]. Founded on these results we enquired whether GOFs mutp53 might be involved in the tumor microenvironment (TME) crosstalk. To this aim we analysed the cytokine secretion profile in a panel of colon and breast human cancer cells, and identified the soluble interleukin-1 receptor antagonist (sIL-1Ra) as a novel mutp53 repressed target gene [6] The sIL-1Ra is a natural occurring anti-inflammatory cytokine that acts as a specific antagonist of the Interleukin-1 (IL-1) $\quad \alpha$ and $\beta$ pro-inflammatory cytokines: it binds to both type I and type II IL-1 receptors (IL-1R1 and IL-1RII), with approximately equal affinity as compared with IL- $1 \alpha$ and IL- $1 \beta$, without exerting any agonist activity. The IL- $1 \beta$ is a highly active and pleiotropic pro-inflammatory cytokine implicated in the pathogenesis of many inflammationassociated diseases. Indeed, recombinant sIL-1Ra (Kineret) is currently used to cure a number of inflammatory and orthopaedic disease. Importantly, the IL-1 $\beta$ expression is found elevated in several human tumors (breast, colon, lung, head and neck, and melanomas), and patients with IL- $1 \beta$ producing tumors have generally bad prognosis.

We found that mutp53 represses whereas activated wtp53 induces sIL-1Ra gene expression uncovering a novel GOF mutp53 [6]. Mechanistically, we identify the MAFF as common molecular player in the opposite regulation of sIL-1Ra gene expression by mut and wtp53 [6]. Indeed, the small MAFs abundance has been identified as a fine tuning molecular switch regulating positively or negatively gene expression. To evaluate the biological significance of sIL-1 Ra suppression in GOF mutp53, we explored the cancer cell response to recombinant IL-1 $\beta$ along with mutp53 depletion or kineret pre-treatment. Results revealed that similarly to pre-treatment with recombinant sIL-1Ra, the derepressed sIL-1Ra in mutp53 depleted cells, hampers the IL-1 $\beta$ signalling cascade by reducing IL-1 target genes expression in vitro and in vivo; the cancer cell proliferation in vitro; and the growth of xenografted tumor in LPS-treated mice. Additionally, the supernatants of either mutp53 depleted or kineret pretreated cancer cells abolishes the IL-1 $\beta$-induced HUVEC endothelial cell monolayer permeability, a hallmark of early angiogenesis [6].

The study shown for the first time the existence of a functional link between sIL-1Ra and mutp53, adding further insights for the identification of novel non-cellautonomous GOFs mutp53 in human cancer. Thus, mutp53 by repressing sIL-1Ra could sustain a prompt IL-1 $\beta$ cancer cell response promoting a chronically inflamed TME, hence fostering further malignancy. Noteworthy, chronic-inflammation is a predisposing cause in various malignancies and is often characterized as the seventh hallmark of cancer [7]. Recent investigations reported that mutp53 sustains cancer progression by augmenting nuclear factor $\kappa \mathrm{B}$ (NFkB) activation in the context of chronic inflammation in 
vitro and in vivo [8]. The $\mathrm{NF \kappa B}$ is a required transcription factor for canonical IL-1 target genes expression. Accordingly, we propose that mutp53 might support a ready-to-be-activated IL-1 signalling cascade in cancer cells through a dual regulatory path: extracellularly by suppressing the sIL-1Ra production, thus reducing the relative protein levels of the receptor antagonist in the microenvironment nearby the cancer cells; - intracellularly increasing the IL-1 target gene expression augmenting NFkB activity.

Remarkably, preclinical studies provide ample support to propose the reduction of IL-1 activity as a potential therapeutic target in human cancers. Accordingly, albeit further investigations are required, achieved results are suggesting that modulation of the TME through the targeting of IL-1 activity combined with currently used chemotherapeutic agents might constitute a novel efficient anti-tumoral strategy for treating mutp53 carrying tumors.

\section{REFERENCES}

1. Muller PA and Vousden KH. Nat. Cell Biol. 2013; 15: 2-8.

2. Lujambio A, et al. Cell. 2013; 153:449-460.

3. Bar J, et al. Oncogene. 2009; 28:933-642.

4. Addadi Y, et al. Cancer Res. 2010; 70:9650-9658.

5. Bossi G, et al. Cell Cycle. 2008; 7:1870-1879.

6. Ubertini V, et al. Oncogene. 2015; 7; 34:2493-2504.

7. Mantovani A. Nature. 2009; 457:36-37.

8. Cooks T, et al. Cancer Cell. 2013; 23:634-646.

Gianluca Bossi: Experimental Oncology Laboratories, and Laboratory of Medical Physics and Expert Systems Regina Elena National Cancer Institute, Rome, Italy

Correspondence: Gianluca Bossi

Email: bossi@ifo.it

Keywords: p53, tumor, microenvironment, inflammation, antagonist receptors, cytokines

Comment on: Mutant p53 gains new function in promoting inflammatory signals by repression of the secreted interleukin-1 receptor antagonist. Oncogene. 2015; 34:2493-504.

Received: September 27, 2015

Published: October 20, 2015 